\section{P241 CONSIDERING PATIENTS' VALUES AND PREFERENCES WHEN FORMULATING RECOMMENDATIONS WITH GRADE}

${ }^{1} \mathrm{~N}$ Ibargoyen-Roteta, ${ }^{1}$ I Etxeandia-lkobaltzeta, ${ }^{2} \mathrm{G}$ Zaragoza-Gaynor, ${ }^{2} \mathrm{~J}$ Carrasco-Gimeno, ${ }^{3} \mathrm{M}$ Lizarraga-Azparren, ${ }^{4} \mathrm{~J}$ Elorz-Lambarri, ${ }^{4} \mathrm{M}$ Villar-Alvarez. 'Basque Office for Health Technology Assessment (OSTEBA), Vitoria-Gasteiz, Spain; ${ }^{2}$ Health and Social Researchers, Madrid, Spain; ${ }^{3}$ Primary Health Centre of Erandio (Osakidetza), Erandio, Spain; ${ }^{4}$ Paediatric Service, Hospital of Basurto (Osakidetza), Bilbao, Spain

\section{0:1136/bmjqs-2013-002293.223}

Background The GRADE approach takes into account the variability that may exist in patients' values and preferences when formulating recommendations. Nevertheless, in the updating process of the Clinical Practice Guideline (CPG) for children with asthma no related information could be found in the literature.

Objectives To obtain information about the values and preferences of caregivers of children with asthma to be considered when formulating recommendations.

Methods A focus group was designed to explore caregivers' perceptions and opinions about some of the issues addressed in the CPG. Twelve possible participants were identified by paediatricians and specialists involved in the development of the GPC, and finally 9 mothers of children with asthma were convened. Twenty-two questions were defined by the research team. The focus group, previous consent recorded, was transcribed and analysed. Triangulation was made by two of the researchers for all the analysis.

Results The information obtained was taken into account in 11 of the 16 clinical questions. The most relevant information obtained was related to the relevance of self-management and the importance of having good information, for caregivers and children, to manage the disease. The concern about what happened when caregivers were not with the children, for example at school, was also identified.

Discussion The focus group helped to obtain information about patients' values and preferences to be considered when formulating recommendations.

Implications for Guideline Developers/Users Focus groups specifically designed to obtain information about patients' opinions in relation to particular clinical questions provides valuable context-dependent information to take into account when formulating recommendations.

\section{P242 MANAGING ETIOLOGY QUESTIONS WITH GRADE}

${ }^{1} \mathrm{~N}$ Ibargoyen-Roteta, ${ }^{1} \mid$ Etxeandia-lkobaltzeta, ${ }^{2} \mathrm{~A}$ Etxeberria-Agirre, ${ }^{3} \mathrm{R}$ Rotaeche-del Campo, ${ }^{4} \mathrm{~A}$ Aldasoro-Ruiz, ${ }^{5} \mathrm{M}$ Lizarraga-Asparren, ${ }^{6} \mathrm{~J}$ Elorz-Lambarri, ${ }^{6} \mathrm{M}$ Villar-Alvarez, ${ }^{7} \mathrm{E}$ Galardi-Andonegui, ${ }^{8} \mathrm{M}$ Irizar-Aranburu. ${ }^{1}$ Basque Office for Health Technology Assessment (Osteba), Vitoria-Gasteiz, Spain; 2 Primary Health Centre of Hernani (Osakidetza), Hernani, Spain; ${ }^{3}$ Primary Health Centre of Alza (Osakidetza), San Sebastian, Spain; ${ }^{4}$ Paediatric Service, Donostia Hospital, San Sebastian, Spain; ${ }^{5}$ Primary Health Centre of Erandio (Osakidetza), Erandio, Spain; ${ }^{6}$ Paediatric Service, Hospital of Basurto (Osakidetza) Bilbao Spain Primary Health Centre of Bidebieta (Osakidetza) San Sebastian Spain Primary Health Centre of Idiazabal (Osakidetza) Idiazabal Spain

\section{0:1136/bmjgs-2013-002293.224}

Background Currently the GRADE approach only considers the development of recommendations for treatment and diagnosis related clinical questions.

Objectives To develop a proposal using GRADE to formulate recommendations for aetiology questions.
Methods The exposure to paracetamol in the first year of life and asthma at the age of 6 years was chosen as an example. A systematic review was made, searching in Cochrane, CRD, Medline and Embase databases. Cohort studies or Meta-analysis of observational studies were selected, and CASPE and MOOSE checklists used for quality assessment. The evidence was considered high when there were no factors affecting our confidence on the estimates.

Results One systematic review (including 4 related cross-sectional studies) and 4 cohorts were found. To assess the evidence with GRADE two outcomes were used (increase of asthma and wheezing at 6 years). Risk of bias was assessed taking into account the cohort design and aetiology studies characteristics, but inconsistency, indirectness and imprecision were considered in the same manner. In contrast to cross-sectional studies, cohort studies (3 out of 4) did not find a significant relationship between paracetamol and asthma development. Due to possible risk of bias and imprecision, the quality of the evidence was downgraded two levels and a recommendation in favour of the rational use of paracetamol in children was made.

Discussion A proposal to address aetiology questions based on the GRADE approach has been developed. More examples are needed to validate it.

Implications for Guideline Developers To be able to use the GRADE approach independently of the question type.

\section{P244 DEVELOPMENT OF A TAILOR-MADE IMPLEMENTATION STRATEGY TO FACILITATE ADHERENCE TO THE DUTCH POSTPARTUM HEMORRHAGE GUIDELINE AND MOET- INSTRUCTIONS}

${ }^{1} \mathrm{~S}$ De Visser, ${ }^{1} \mathrm{M}$ Woiski, ${ }^{2} \mathrm{M}$ Hulscher, ${ }^{1} \mathrm{~F}$ Vandenbussche, ${ }^{3} \mathrm{~L}$ Scheepers, ${ }^{2} \mathrm{R}$ Hermens. ${ }^{1}$ Radboud University Nijmegen Medical Centre, Department of Obstetrics \& Gynaecology, Nijmegen, The Netherlands; ${ }^{2}$ Radboud University Nijmegen Medical Centre, Department of IQ Healthcare, Nijmegen, The Netherlands; ${ }^{3}$ Maastricht University Medical Centre, Maastricht, The Netherlands

\section{0:1136/bmjqs-2013-002293.225}

Background Despite the introduction of a national guideline and the Managing Obstetric Emergencies and Trauma (MOET) course the incidence of postpartum haemorrhage (PPH) is increasing. We suspected inadequate guideline implementation to be underlying.

Objective Our objective was to develop and pilot test a tailormade implementation strategy for the Dutch PPH guideline and the MOET course to increase guideline adherence.

Methods Firstly, we aggregated the results of a current care and diagnostic analysis, which yielded barriers for guideline implementation, together with evidence found in international literature. Main barriers among professionals were lack of a checklist available in the labour ward, inadequate high-risk patient identification and lack of team communication. Patients mentioned insufficient information supply. Secondly, we developed different implementation tools for professionals and patients, based on these results. These tools were evaluated in a pilot testing among both groups.

Results The strategy affects the period between the last trimester of the pregnancy till the end of the third stage of delivery. The tools for professionals consisted of a high-risk identification checklist, a care bundle for PPH prevention, and a PPH treatment checklist. As patient empowerment tools a patient passport and an information website were developed. Professionals had 